\title{
Free-Electron Lasers Push into New Frontiers
}

\author{
Stephen V. Benson \\ Thomas Jefferson National Accelerator Facility (Jefferson Lab) \\ 12000 Jefferson Avenue, Newport News, VA 23606
}

\begin{abstract}
From the early days of the development of free-electron lasers (FELs) the promise of high power and short wavelengths has tantalized physicists and other scientists. Recent developments in accelerator technologies and some new discoveries about the physics of FELs have allowed researchers to push the performance of FELs into new frontiers of high power, short wavelength, and ultra-short pulses. Spin-offs from the FELs have also opened up new radiation sources in the THz, X-ray and gamma ray wavelength ranges.
\end{abstract}

\section{INTRODUCTION}

The free-electron laser is an attractively simple device. An electron accelerator produces a beam of electrons that is sent through a wiggler - a magnet whose field varies sinusoidally with distance. The electrons radiate and bunch at a resonant wavelength determined solely by the wavelength of the wiggler, the wiggler magnetic field, and the electron beam energy. The resulting radiation may build up to saturation in a single pass or be captured in an optical resonator and rise to saturation in an oscillator (see Figure 1).

Due to their inherent simplicity, the theory of FELs was well developed early in their history. The physics and technical status of FELs is covered by several review articles $^{1-3}$ to which I refer the interested reader. This paper concentrates on recent accomplishments in the field that have allowed laser builders to push the capabilities of the FEL into new regimes of power, wavelength, and pulse length.

From the earliest days of FELs, the possibility of providing light at wavelengths inaccessible to light sources based on electrons bound in atoms or molecules and of providing very high average power was clear. Since the FEL wavelength is not tied to an existing atomic or molecular transition, the resonant wavelength can be tuned from 
millimeters to nanometers. Since the unused energy in the electron beam leaves the laser at nearly the speed of light, the devices can, in principle, provide extremely high average power as well. Early devices had low gain, however, and generally lased at wavelengths in the infrared with power output on the order of $1 \mathrm{~W}$. This article describes improvements to electron beams and the improvements to FEL performance that have followed.

\section{Improvements in Electron Beam Quality}

Due to the quality of the available electron beams, two decades passed between the first demonstration of the FEL concept and the demonstration of short wavelengths and high average power. Though early accelerators could produce megawatts of low quality electron beam, energies high enough to reach the X-ray region, and very high quality electron beams at low peak and average current, they could not produce the high peak current and excellent beam quality necessary for a short wavelength or high power FEL.

The most important technological leap for short wavelength FELs was the development of the photocathode electron gun ${ }^{4}$. Photocathode electron guns have produced very high quality electron bunches that can be compressed down to sub-picosecond pulses with kilo-ampere peak currents, thus greatly enhancing the gain at short wavelengths. Such high quality beams allow the spontaneous radiation to build quickly to saturation in a single pass in a so-called self-amplified spontaneous emission (SASE) type of FEL. This allows operation in wavelength ranges where no high reflectivity mirrors are available.

The most important new technology for high average power was the energy-recovering accelerator using superconducting radio frequency (SRF) accelerating cavities. Energy recovery of the electron beam and the use of SRF acceleration, coupled with the use of a photocathode electron gun, have permitted the efficient production of extremely high average power electron beams with the quality required for an FEL. The energy-recovering accelerator now in development at Jefferson Lab will feature over $1 \mathrm{MW}$ of continuously circulating electron beam.

\section{Electron Beam Brightness}

The quality of the electron beam for an FEL is measured by the brightness, which is proportional to the electron bunch charge divided by the six-dimensional phase space volume occupied by the electrons in the bunch. This phase space volume (the product of the transverse dimensions, the transverse velocities, the bunch length, and the energy spread) must be small enough to allow high peak current while the energy spread and emittance are less than those acceptable for the FEL. The geometric emittance of the electron beam (the product of the rms beam size and angular spread) must be less than the optical wavelength divided by $4 \pi$. Clearly, this becomes more difficult for short 
wavelength devices. For an oscillator, the rms energy spread must be less than $1 /\left(5 N_{W}\right)$, where $N_{W}$ is the number of wiggler periods. For an amplifier, the energy spread must be less than $1 / 7 \pi$ times the gain per wiggler period. Many electron beam instabilities tend to increase the geometric emittance and the energy spread, so it is not only necessary to create a bright electron beam, it is also necessary to preserve the brightness as the electron beam is accelerated and transported to the laser. Great progress has been made in the last decade in both creation and transport of bright electron beams.

The brightest beams are produced in pulsed RF photocathode guns. These embed the photocathode in a high performance room temperature RF cavity with a field gradient greater than $100 \mathrm{MV} / \mathrm{m}$. A high-density electron pulse is accelerated to as much as 5 million volts in a few centimeters. The electrons become fully relativistic before the electrostatic repulsion between them can ruin the beam quality. Once they are fully relativistic, the electrostatic repulsion in the lab frame is greatly reduced, allowing the beam to be transported with little further degradation.

One can also make a photocathode gun with a DC cathode. Since the voltage on the cathode is on all the time, the maximum sustainable gradient is much less than in a pulsed RF gun. Nevertheless, reasonably bright beams can be produced with high average current. The average brightness is much higher than the pulsed sources and is more than sufficient for a high average power FEL. The peak brightness is not sufficient at present to drive a short wavelength SASE FEL.

Recently researchers at Rossendorf Research Center near Dresden, Germany, in collaboration with the Budker Institute in Novosibirsk, Russia, have developed a superconducting RF photocathode gun ${ }^{5}$. Though in its infancy, this source might combine the best aspects of pulsed RF and DC photocathode guns. If so, one might consider using one in a high power SASE FEL.

\section{High Average Electron Beam Current}

The most important parameter for a high average power FEL is the average electron beam current. High beam current permits the generation of MW electron beams. Since FELs typically extract only a few percent of the power from an electron beam it makes sense to try to recover some of the electron beam power instead of throwing it away. One way to do this was proposed long before the invention of the FEL in 1965 by Maury Tigner at Cornell University. His idea was to decelerate the electron beam in the same cavities used to accelerate the beam. Dumping the beam at nearly the full energy leads to very high radiation and severe thermal management issues. If the electron beam can be decelerated in the same cavities in which it was accelerated, the problems with dumping the beam become quite manageable, the RF power requirements for the accelerator are lessened tremendously, lowering the system cost, and 
the overall efficiency of the laser is increased. Dumping a beam at low energy eliminates the production of radioactive beamline components. The lack of residual radiation in the dump is an especially important issue for industrial acceptance.

The second challenge in accelerating high average current is beam loss. Electron beam loss at any one point must be kept to less than $100 \mathrm{nA}$ out of a total current of up to $10 \mathrm{~mA}$. This is extremely good transport efficiency. We have found however that the DC photocathode source provides a very clean electron beam. Beam losses even at points where the chamber necks down are typically less than $1 \mathrm{nA}$ when running at $5 \mathrm{~mA}$. The total losses at high energy are less than $100 \mathrm{nA}$.

The final challenge posed by high average current is the initiation of regenerative beam instabilities. These instabilities are caused by parasitic accelerating modes that can be driven by imperfections in the electron beam position or energy. These instabilities were found in the first recirculating accelerators and great progress has been made in understanding and alleviating them ${ }^{6}$. The first defense against these instabilities is to damp the offending accelerating modes. This can raise the threshold for instability to tens of mA. Note that the dampers must be carefully designed to handle the power from these modes, which may easily exceed one watt per mA of beam current. Feedback might be used to further increase the instability thresholds to the order of $1 \mathrm{~A}$ of electron beam current.

\section{Towards Shorter Wavelengths}

It was realized early in the development of FELs that self-amplified spontaneous emission, already demonstrated in conventional lasers, might be a way for FELs to operate in wavelength ranges where high reflectivity mirrors do not exist. With measured gains of a few percent per meter, however, the development of a SASE FEL awaited the development of a brighter electron source. In 1992, Pellegrini proposed using a recently developed RF photocathode electron gun coupled to the two-mile Stanford linear accelerator to produce lasing in the hard X-ray region ${ }^{7}$. The facility was dubbed the Linac Coherent Light Source (LCLS). The realization that such a source might provide the technology for a new generation of X-ray sources led many groups to start development of SASE devices in the visible to vacuum ultraviolet (VUV) spectral ranges to demonstrate the concept and develop the technologies necessary to build linear light sources.

After many years of development, efforts to produce saturated lasing using a SASE FEL started to come to fruition in the start of this century. In September 2000 a group at Argonne National Lab (ANL) became the first to demonstrate saturation in a visible SASE FEL. Since then they have pushed the device into the vacuum ultraviolet at $150 \mathrm{~nm}$. The 
VISA collaboration between Brookhaven National Lab (BNL), UCLA and The Stanford Linear Accelerator Center (SLAC) has demonstrated lasing to saturation at $800 \mathrm{~nm}$ with a very short wiggler only $4 \mathrm{~m}$ in length. This project was designed to test the possibility of scaling the technology to the hard X-ray range. Recently the Department of Energy, encouraged by these results, has given SLAC the go-ahead for engineering design of the LCLS device to be constructed at SLAC (see figure 2). This device should produce $50 \mathrm{fsec} \mathrm{X}$-ray pulses with over $20 \mathrm{GW}$ of peak power. Such a short, high power X-ray pulse may be able to take holographic images of complex bio-molecules with one pulse before the high intensity has had a chance to completely destroy the molecule. There are now at least eight serious proposals for SASE FEL facilities worldwide operating in the VUV to hard X-ray wavelength regime. This is quite a remarkable state of affairs when one considers that no device had even lased to saturation in the visible as late as the summer of 2000.

Most SASE devices being developed are proof-of-principle devices that will support only a small number of simultaneous users. The Tesla Test Facility FEL (TTF FEL) is a system based on superconducting radio frequency (SRF) acceleration that is heading directly toward a user facility. It is now in commissioning at DESY (Hamburg, Germany) and has already lased to saturation at $98 \mathrm{~nm}$, the shortest wavelength at which any FEL has saturated. It has the prospect of producing high repetition rates. While SRF technology is not required to reach short wavelengths, its use is essential for a Next Generation User facility to achieve the duty factor necessary to service many users. The concept is to continuously switch the beam among a farm of wigglers with a user experiment at the output of each. Recently the German government approved funding for an upgrade to this facility to produce a hard X-ray FEL user facility.

Not all short wavelength FELs are SASE devices. In 1999 a BNL/ANL/UCLA collaboration showed lasing to saturation using a sub-harmonic seed laser $^{8}$. This technique, called High Gain Harmonic Generation (HGHG), relies on the fact that FELs emit harmonics of the lasing wavelength. Furthermore the harmonics are preserved in the electron density distribution and can be used to seed a harmonic amplifier in the form of a second wiggler tuned to one of the harmonics. The seed laser for an amplifier can thus be at a subharmonic of the eventual laser frequency. This technique is now being looked at as a way to enhance the spectral purity of radiation from soft X-ray FELs. As an example, one might seed an FEL amplifier at $157 \mathrm{~nm}$ using a fluorine laser but have a downstream wiggler lase at a wavelength of 31 $\mathrm{nm}$. This radiation might then be used to seed another amplifier at $6.3 \mathrm{~nm}$. The advantages of this approach are that the output radiation is much more coherent than a SASE device and the pulse-to-pulse fluctuations are greatly reduced. Recently the Brookhaven group extended the HGHG technique to the UV. They plan to operate in the VUV shortly. 
The spectrum of the output from the HGHG device and the output of the same device operated in SASE mode are shown in figure 3. The pulses from the harmonic generation device are nearly transform-limited.

\section{Towards Higher Average Power}

In the late 1990s our group at Jefferson Lab used the idea of same-cell energy recovery to build an accelerator with $5 \mathrm{~mA}$ of electron beam at $48 \mathrm{MeV}$. When sent through a wiggler, the resulting $230 \mathrm{~kW}$ electron beam produced over 2 $\mathrm{kW}$ of laser light at $3 \mu \mathrm{m}$. The efficiency of this device was not optimized since it was just a proof-of-principle device. Nevertheless, the wall plug efficiency proved to be $0.5 \%$.

More recently our group has been commissioning the successor to the $2 \mathrm{~kW}$ machine. The current is being raised by a factor of two to $10 \mathrm{~mA}$. The energy is being raised by a factor of more than 3 to over $150 \mathrm{MeV}$, leading to a circulating electron power of 1.5 MW. The extraction efficiency should also increase by at least $50 \%$. The power should therefore increase by a factor of 10 to over $20 \mathrm{~kW}$ in the mid-infrared wavelength range. The wall plug efficiency should rise to as much as $2 \%$, again with no system optimization for efficiency. Future upgrades of this system promise over $6 \%$ wall plug efficiency when operated at $100 \mathrm{~kW}$. The fast rise in efficiency with increasing power is due to large power requirements that are independent of electron beam current such as the main accelerator RF, the cryogenic refrigerator, and the magnet power supplies. Only the injector drive power changes with current.

The new system is shown schematically in figure 4 . The electron beam is created in a $350 \mathrm{kV}$ DC photocathode gun illuminated by a frequency-doubled, mode-locked Nd:YLF laser. The beam is then accelerated up to $10 \mathrm{MeV}$ in a pair of superconducting accelerating cavities and merged into the main accelerator, also superconducting, which uses three eight-cavity accelerating modules to accelerate the beam to over $150 \mathrm{MeV}$. The beam is then transported around the high reflector mirror of the optical resonator, and sent through the $6 \mathrm{~m}$ long wiggler at the center of a $32 \mathrm{~m}$ long nearly concentric resonator. The electron beam is then transported back to the accelerator, merged with the injector beam, and injected into the accelerator at a decelerating phase so that its energy is reduced to the original injection energy of $10 \mathrm{MeV}$. The beam is then separated from the accelerated beam and dumped. Since the main accelerator both accelerates and decelerates the electrons, only the power necessary to keep the accelerating cavities at the operating voltage is needed. Since the cavities are superconducting, very little power is required to keep them at the operating voltage. This fact, and the high continuous voltage available from superconducting accelerating cavities, makes them ideal for energy-recovering accelerators. 
The accelerator will also be used to drive a lower power FEL in the ultraviolet. The UV demonstration laser shown in figure 4 should produce more than $1 \mathrm{~kW}$ at wavelengths as short as $250 \mathrm{~nm}$ and several $\mathrm{kW}$ at wavelengths near 350 nm. To operate the UV laser, one merely changes the two switch magnets to send the laser into the outer electron beam transport. The energy recovery works the same as with the IR laser. The $2 \mathrm{~kW}$ infrared laser provided a quantum leap in available power in the near infrared to visible range by providing over $300 \mathrm{~W}$ of tunable picosecond pulses at 1.06 microns $56 \mathrm{~W}$ at $530 \mathrm{~nm}$ (see figure 4) and $14 \mathrm{~W}$ at $355 \mathrm{~nm}$. This is far more than any other ultrafast laser has demonstrated. The upgraded laser will be another quantum leap in performance, providing IR to UV light with two orders of magnitude more power and with even shorter pulses.

The infrared upgrade FEL at Jefferson Lab will test many new technologies designed to allow future advances to even higher power. Higher voltage standoff in the photocathode gun should allow brighter electron beams to be produced. This will allow higher efficiency and/or shorter wavelengths to be produced. The electron beam transport system was intentionally over-designed to allow higher efficiency from the FEL and higher current from the injector. The primary power limitation factor will then be the mirrors of the optical resonator. The present nearly concentric resonator is very sensitive to heating-induced mirror deformation. We plan to test new technologies such as low loss mirror coatings, deformable mirrors, and cryogenic mirrors to extend the power limits to beyond $100 \mathrm{~kW}$ in the near infrared.

Advances in the realm of beam transport and superconducting RF cavities should also reduce the size and complexity of the energy-recovery accelerator. One accelerating module now has the accelerating voltage of two older modules. Cavities in development at other labs have demonstrated another factor-of-two improvement.

High current FELs with energy recovery have some interesting features. When very short electron bunches are bent in a magnet they radiate strongly at all wavelengths longer than the electron bunch. For electron pulses in the new machine at Jefferson Lab, this means that the electron beam radiates strongly at all wavelengths longer than around $100 \mu \mathrm{m}$. This is the $\mathrm{THz}$ wavelength range, which has garnered a great deal of interest lately. Because the electrons are relativistic, they radiate much more strongly than the electrons in semiconductor $\mathrm{THz}$ sources. The result is that over $100 \mathrm{~W}$ of $\mathrm{THz}$ radiation should be produced from the electron beam entering the FEL. Note that we do not have to do anything special to the electron beam beyond making it the right size and length for lasing. The THz radiation is then automatically optimized. Similarly, when the electrons collide head-on with circulating photon bunches, some of the photons scatter off the electrons. The resulting photon energies are in the hard X-ray range with energies up to several $\mathrm{MeV}$. Though not nearly as bright as a typical synchrotron light source, the X-rays produced from this source are 
monochromatic and come in sub-picosecond pulses. Again, nothing special has to be done to produce these X-rays. Their production is optimized whenever the laser is optimized. At Duke University in Durham NC a similar scheme is used to produce a bright source of gamma rays for nuclear physics research ${ }^{9}$.

Of course an FEL can also be used directly for applications. Power in excess of $10 \mathrm{~kW}$ is of interest to industry if it can be produced for low enough cost. The scaling of the power in infrared free-electron lasers indicates that a $100 \mathrm{~kW}$ device should be cost effective for many industrial processes such as laser glazing, pulsed laser deposition, surface nitriding, or surface texturing. The lower power UV FEL will allow many laser processing techniques already demonstrated with excimer lasers. The cost of the photons may eventually be lower than those of excimer lasers and the wavelength may be tuned to an atomic resonance to enhance certain processes.

\section{Towards Shorter Pulses}

Even early FELs generally emitted picosecond laser pulses. Experimental and theoretical progress made in the 1990s has enabled FELs to produce much shorter pulses. Present FELs can produce pulses only a few wavelengths in length. New understanding of what is required to produce such short pulses may allow the production of extremely short pulses in the near future. A new development in FEL theory has been the behavior of lasers operating at perfect synchronism. In any FEL oscillator driven by an RF linear accelerator, one must match the round trip time of the photons in the resonator with the arrival time of the electron bunches. Previous FEL theory showed that the peak in the power vs. cavity length is always slightly shorter than the length for perfect synchronism. Recent simulations and experiments at the Japan Atomic Energy Research Institute in Tokai, Japan, indicate that, for sufficiently large gain, the peak in the power curve occurs exactly at perfect synchronism ${ }^{10}$. In addition, the pulses in this mode of operation are extremely short and the efficiency is very high. The JAERI group demonstrated laser pulses at $23 \mu \mathrm{m}$ with a FWHM of less than 260 fsec. An autocorrelation trace of the laser pulses when the cavity length was at perfect synchronism is shown in figure 6. The inferred pulse shape is less than 3.5 optical periods in length.

The requirements of the FEL to produce even shorter pulses are the same as those for high average power and short wavelengths, i.e. high peak current and high quality electron pulses. When high quality electron bunches are produced with a bunch length less than the product of the number of wiggler periods and the optical wavelength, and when the resonator losses are low, the laser pulse at perfect synchronism can be much less than the electron bunch length. Using this technique it should be possible to produce pulses less than $10 \mathrm{fsec}$ in the near infrared and visible. This has been demonstrated already using ti:sapphire laser oscillators, but in this case, the radiation will be in the form of a tunable 
multi-kilowatt laser beam. Using an internal focus it may be possible to focus the resulting terawatt of circulating peak power down to reach a peak intensity of over $10^{19} \mathrm{~W} / \mathrm{cm}^{2}$ with a duty cycle greater than $10^{-7}$ (compared to $<10^{-10}$ for a state-of-the-art tabletop terawatt laser).

It is also possible to use standard chirped pulse techniques to produce longer pulses and compress them outside the laser to ultra-short pulses. It may be possible to use this technique in a high gain soft X-ray amplifier to produce subfemtosecond pulses. Such short pulses may be focused down enough to produce high enough fields to cause the vacuum itself to break down.

\section{CONCLUSION}

Though large and expensive, FELs are showing that they can be quite useful devices. The flexibility of an electron gain medium allows FELs to be adapted to many applications that are impossible to achieve with any other technology. Advances in electron accelerator technology and FEL physics and technology have enabled FELs to fulfill some of the promise that was apparent at their discovery. In the future, FELs may be a major source of photons for X-ray light sources and industrial applications.

Sidebar:

\section{Extending the SASE Concept}

Most SASE FEL projects use a simple design with a uniform wiggler at one wavelength. Many groups are looking at ways to extend the performance of these designs. Harmonics are being explored as a way of extending the capabilities of both amplifiers and SASE devices. I have already mentioned the HGHG concept of seeding at a long wavelength to get more coherent output in the soft X-ray range. Variations of this technique are already being explored to extend the technique to the hard X-ray region. As noted above, a SASE FEL at saturation will emit radiation at the harmonics. The power in these harmonics may exceed $1 \%$ of the fundamental power. This may be an inexpensive way of getting very short wavelengths from SASE FELs. Several schemes have been proposed to further enhance these harmonics. At the end of the first wiggler one can put a wiggler that is resonant at a harmonic of the resonant wavelength of the first wiggler. This is reminiscent of the HGHG technique described above but does not require a seed laser. Dattoli has proposed building harmonics into the original wiggler to enhance the harmonic gain. Finally, several 
ways are being explored to produce even shorter pulses from the SASE FELs. Many experimenters would like to have pulses as short at $20 \mathrm{fsec}$. Pulse compression or pulse slicing may be used to reach this pulse length.

Harmonic emission is not limited to amplifiers. Oscillators also emit harmonics and researchers at Duke University recently extracted the third harmonic of their storage ring based oscillator FEL operated in pulsed mode. This technique may eventually provide reasonably bright pulsed coherent light in the soft X-ray region.

End of sidebar. 


\section{ACKNOWLEDGEMENTS}

Many thanks go to those who contributed information and figures to this article. Jaynie Martz did the illustrations for

figures 1 and 4. This work was supported by U.S. DOE Contract No. DE-AC05-84-ER40150, the Office of Naval Research, the Commonwealth of Virginia and the Laser Processing Consortium.

\section{REFERENCES}

1. W. B. Colson, E. D. Johnson, M. J. Kelley, and H. A. Schwettman, 2002 Physics Today, January, 2002, 35.

2. H. P. Freund and P. O'Shea, 2001, Science 292, 1853.

3. H. P. Freund and G. R. Neil, 1999, Proc. IEEE Vol. 87, No. 5, 782.

4. C. Travier, 1991, Nucl. Inst. and Methods in Phys. Res., A304 285.

5. Jansen, D. et al., 1997, Proc. of the 1997 Particle Accelerator Conf., Vancouver, Canada.

6. L. Merminga, 2002, Nucl. Inst. And Meth. in Phys. Res., A483 107.

7. C. Pellegrini, 2001, Nucl. Inst. And Meth. in Phys. Res., A475 1 provides an excellent summary of SASE FELs.

8. Yu L. H., et al., 2000, Science 289, 932.

9.N. Pietralla et al., 2002, Nucl. Inst. And Meth. in Phys. Res., A483 556.

10. R. Hajima, N. Nishimori, R. Nagai, and E. J. Minehara, 2002, Nucl. Inst. And Meth. in Phys. Res., A483 113. 


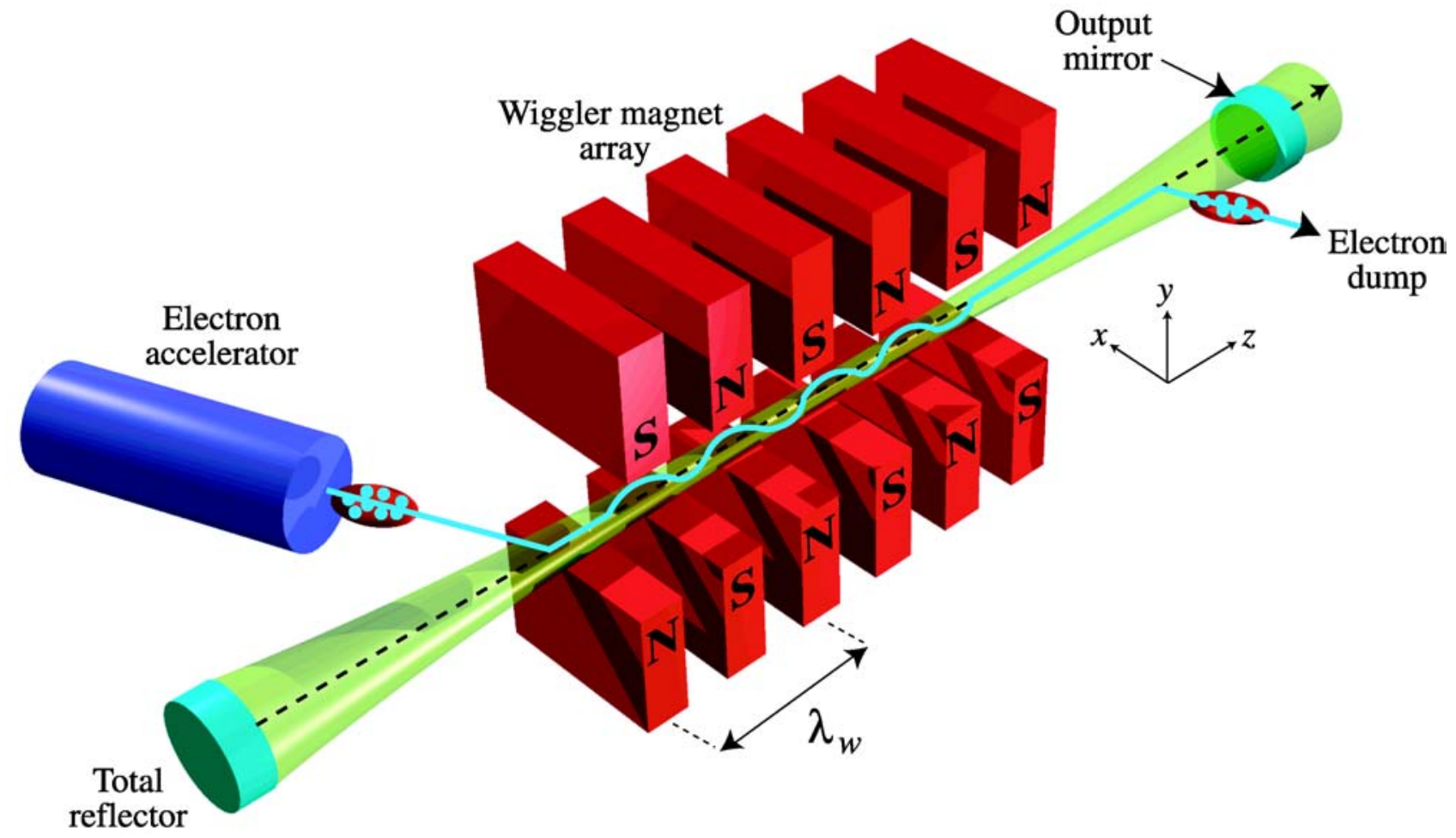

Figure 1: Components of a free-electron laser oscillator. 


\section{The LCLS \\ (Linac Coherent Light Source)}

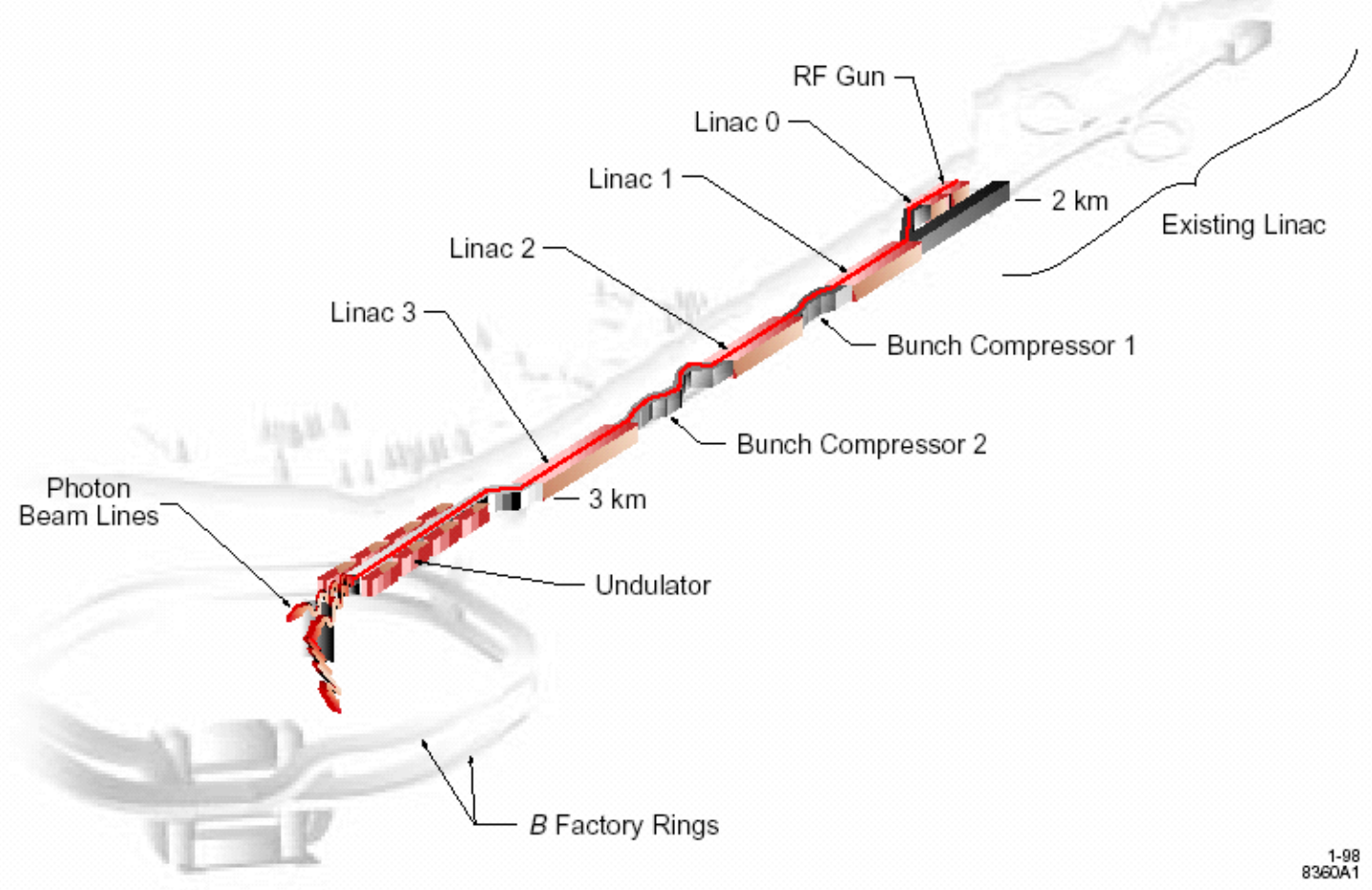

Figure 2. Schematic view of the Linac Coherent Light Source to be built using part of the two-mile accelerator at the Stanford Linear Accelerator Center. 


\section{Spectrum of HGHG and SASE at $266 \mathrm{~nm}$}

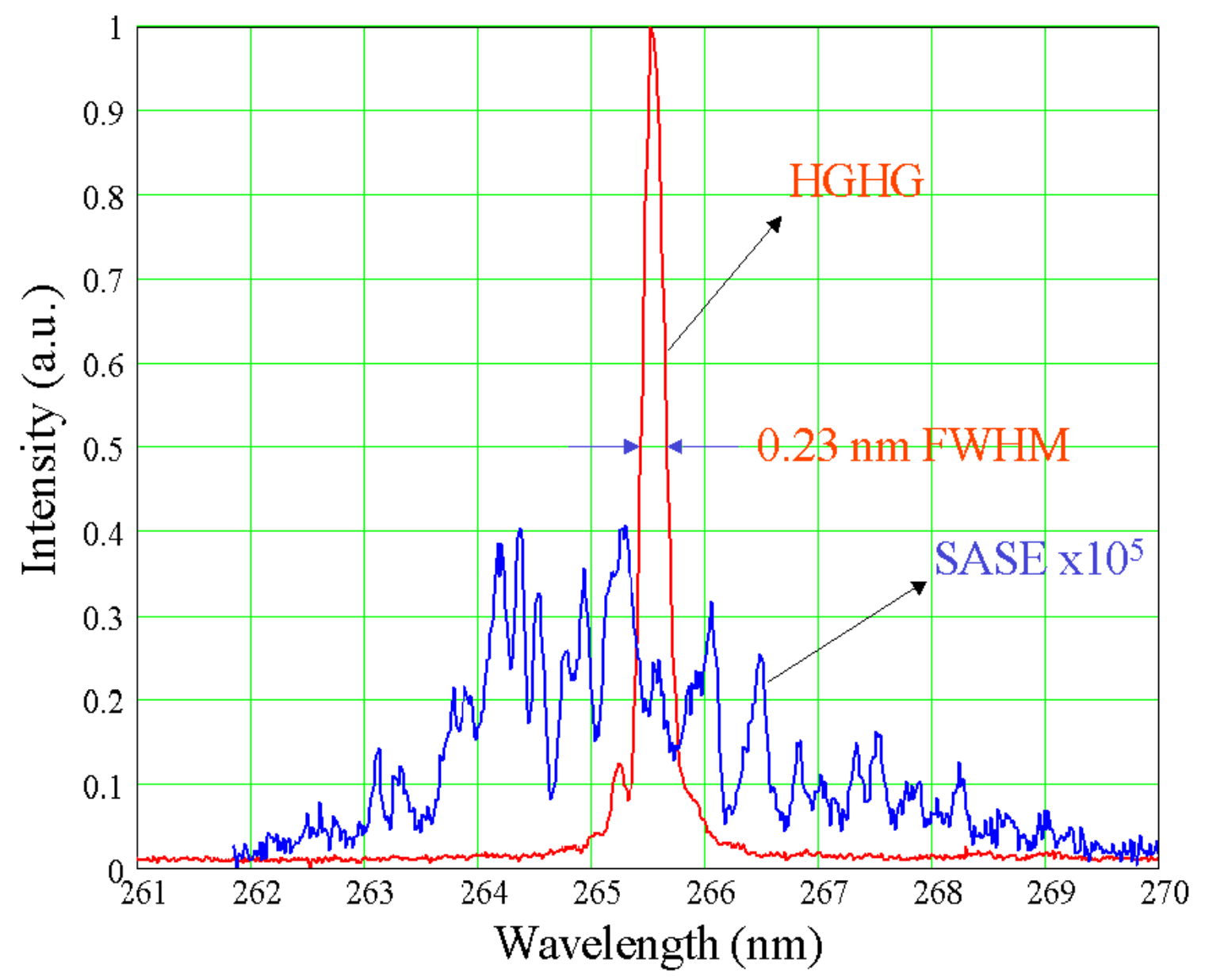

Figure 3. Comparison of FEL operating in the High Gain Harmonic Generation mode and in Self-Amplified Spontaneous Emission mode. The HGHG spectrum is nearly transform limited. The SASE spectrum is noisy and very broad. The HGHG pulse is $150 \mu \mathrm{J}$ in a single pulse. 


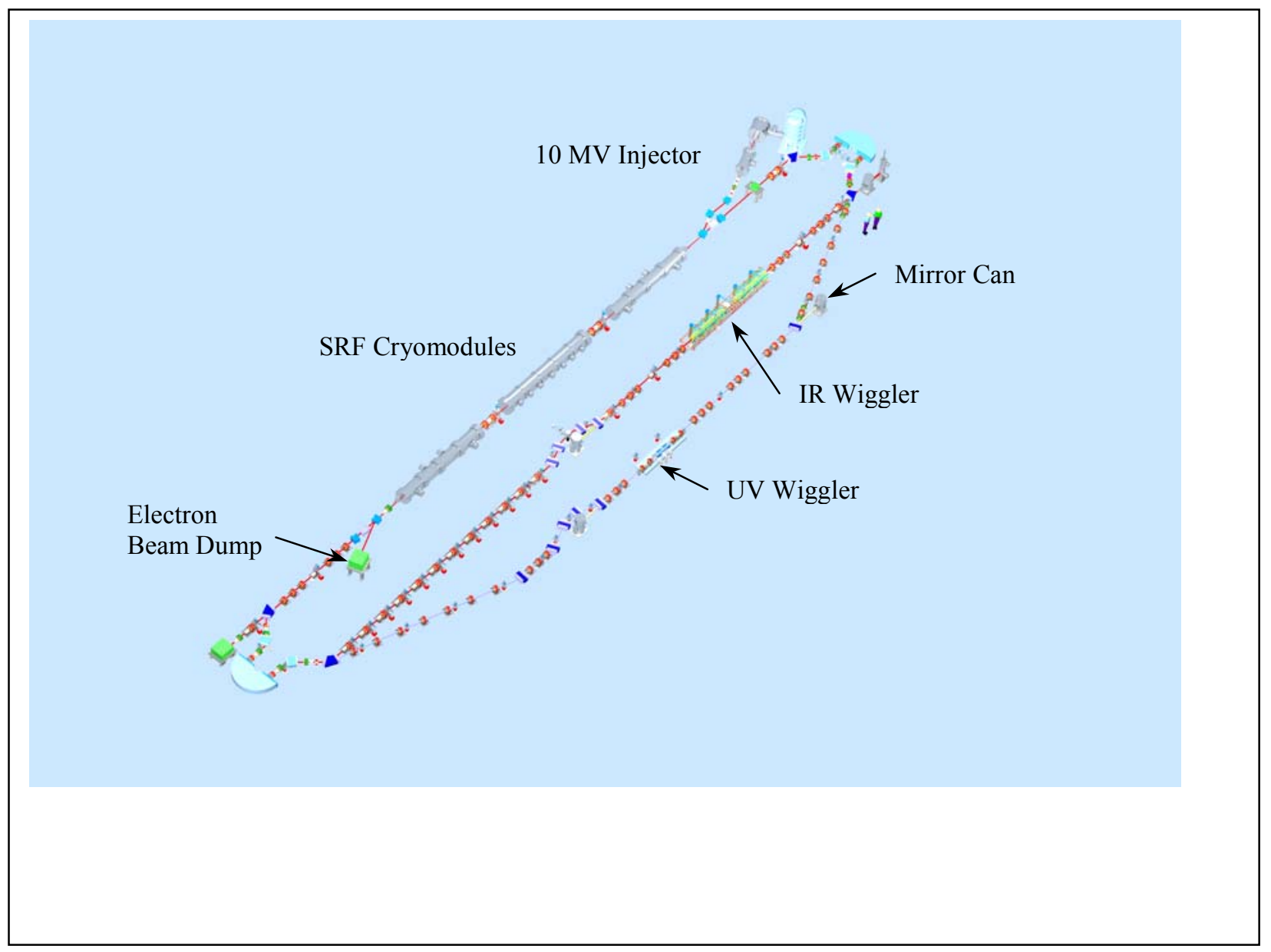

FIG. 4. Schematic layout of the $10 \mathrm{~kW}$ infrared and $1 \mathrm{~kW}$ UV laser at Jefferson Lab. IR laser is on the inner track. The UV laser is on the outer track. 


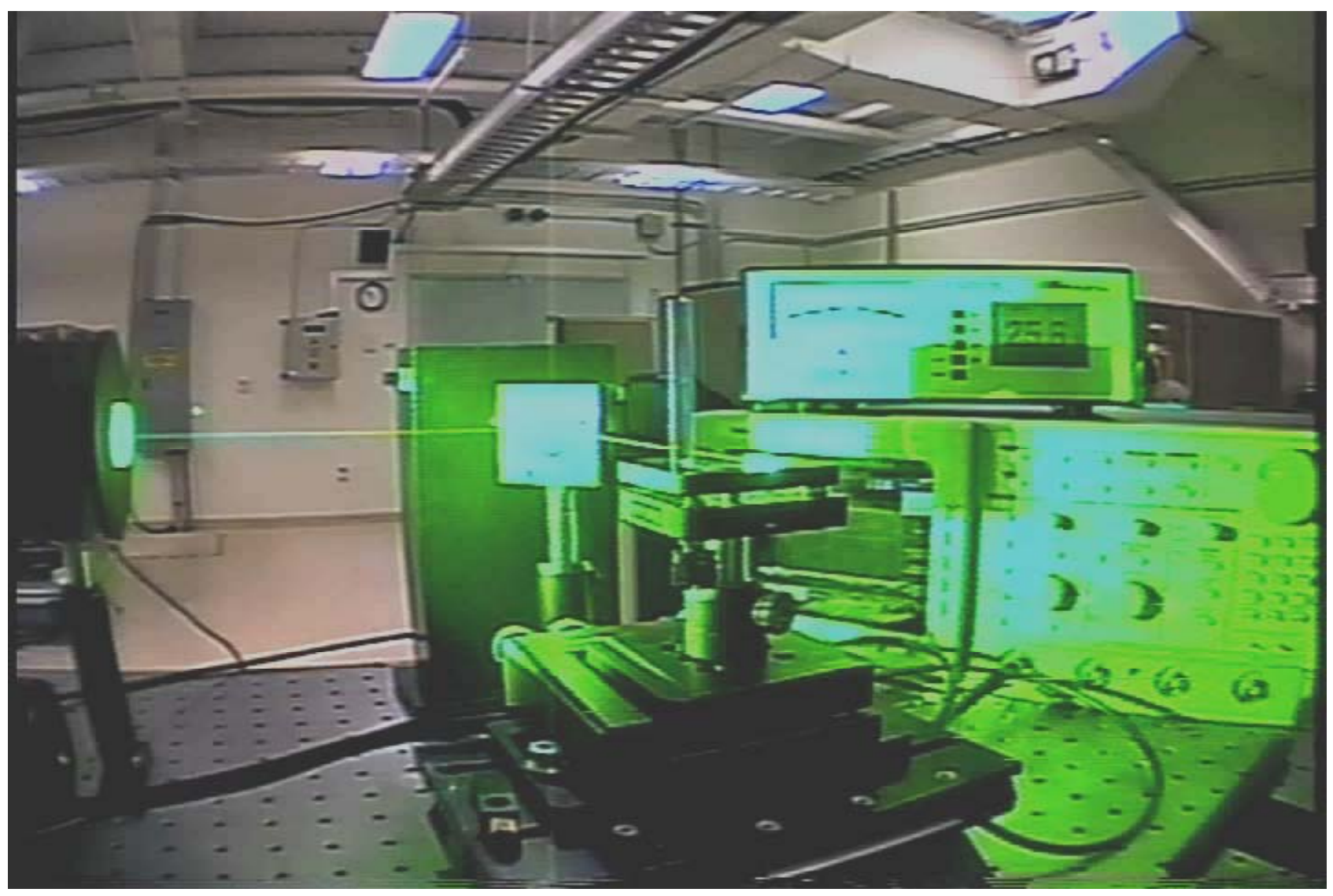

Figure 5. The $2 \mathrm{~kW}$ FEL at Jefferson Lab was the highest demonstration of picosecond pulses at 1 micron from any laser to date. It produced over $300 \mathrm{~W}$ of picosecond pulses at 1.06 microns. Here a part of that 1 micron light is being doubled in an LBO crystal. Over $50 \mathrm{~W}$ of picosecond pulses at $530 \mathrm{~nm}$ could be produced in this way. The upgraded system will lase directly in the green to ultraviolet and provide several kilowatts of green with subpicosecond pulses. 


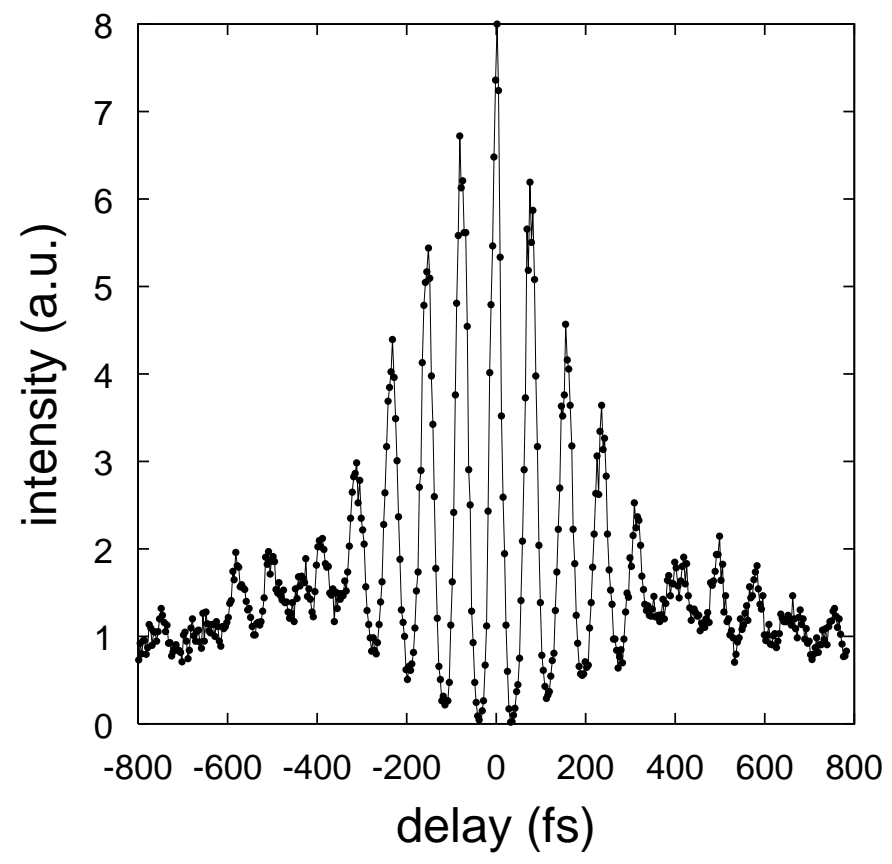

FIG. 6. Autocorrelation signal from the JAERI FEL operated at perfect synchronism. The FWHM pulse length, assuming $\operatorname{a~sech}^{2}(\mathrm{t})$ profile, is $255 \mathrm{fsec}$. 